\title{
New Microfluidic Paths to Test for Bleeding or Clotting
}

\author{
Scott L. DiAmond \\ Department of Chemical and Biomolecular Engineering, Institute for Medicine and Engineering, University of Pennsylvania, \\ Philadelphia, PA 19104, USA \\ (Published online 18 January 2017)
}

The medical terms "thrombosis" and "hemostasis" are used so frequently to describe blood coagulation that it is easy to forget the difference. Hemostasis involves blood leaving the vasculature compartment, while thrombosis involves blood clotting inside the vascular compartment. The fluid mechanics, ${ }^{1}$ pressure dynamics, and intrathrombus transport physics ${ }^{3}$ are unique to each situation. Relevant to diverse clinical scenarios of heart attacks, strokes, deep vein thrombosis, safe anticoagulation, medical device performance, and traumatic or hemophiliac bleeding, the past 10 years has produced several new microfluidic approaches to study blood function. ${ }^{2,4}$

This Issue announces two new devices to study blood under flow. Schoeman et al. ${ }^{5}$ describe a new way to measure "hemostasis on a chip" where blood can leave a high pressure flow channel until the bleeding passageway supports a hemostatic clot. The new design required careful consideration of the pressure dynamics in the hydraulic circuit so that blood could escape a higher pressure flow channel into a lower pressure receiver channel. The "injury channel" was coated with procoagulants, collagen and tissue factor, that allowed healthy blood to form a hemostatic clot to stop on-chip "bleeding" in $7.5 \mathrm{~min}$. The wall shear rate in the injury channel was about $10^{4} \mathrm{~s}^{-1}$, which is quite high and potentially sufficient to evoke changes in von Willebrand Factor (VWF) structure/function. The 150$\mu \mathrm{m}$ long hemostatic clots that formed were rich in both platelets and fibrin and withstood transclot pressure drops of $\sim 1 \mathrm{kPa}(7.5 \mathrm{~mm} \mathrm{Hg})$. The chip was able to phenocopy a genomic condition and a pharmacological condition. Defects in blood to mimic hemophilia A (via anti-Factor VIII antibody) resulted in an unstable hemostatic clot. Treatment of blood with antiplatelet $\mathrm{P} 2 \mathrm{Y}_{12}$ receptor inhibitor substantially delayed

Address correspondence to Scott L. Diamond, Department of Chemical and Biomolecular Engineering, Institute for Medicine and Engineering, University of Pennsylvania, Philadelphia, PA 19104, USA. Electronic mail: sld@seas.upenn.edu the closure time. Novel applications of this device include the testing of new recombinant proteins or nanoparticles as hemostatic agents for prophylactic or emergency use, either in healthy human blood or patient blood. Similarly bleeding risks associated with off-target drug action on platelets could be evaluated with this new design.

Zilberman-Rudenko et al. ${ }^{6}$ explored a related flow geometry, the microfluidic ladder, to investigate thrombotic processes in blood. While several groups continue to use straight channels to study blood clotting on a surface at uniform wall shear rate, the ladder geometry provides several separate rung locations for flow branching. Blood flow enters the ladder along one rail and then must cross sequential rungs of the ladder after which the blood is then collected along the opposing rail. A coating of the device interior with collagen and tissue factor was used to drive clotting. As one rung clots fully, flow is diverted to the other rungs. The clotting was predicted based on particle tracking simulations to occur preferentially at the branch points, a result verified by direct visualizations on the device. While boundary layer separation and reattachment and flow vortexes can exist in vivo distal of severe coronary stenosis, such hemodynamics are difficult to recreate at the low Reynolds numbers typical of microfluidics. Still, flow over a dead end cavity (the proximal end of the exit ladder rail) allowed a recirculation flow with consequent prolonged residence times and propensity for clotting. Clotting in recirculating cavity flows is particularly relevant to the hemodynamics within extracorporeal or implanted device geometries as well as within aneurysms.

These two new devices add to the growing armory of microfluidics to study flowing blood under coagulating conditions. The incorporation of endothelium into the operation of these devices remains a future possibility. Beyond their utility in research, these devices may exist someday at the patient bedside or in the coagulation lab to assist clinical decision making. 


\section{REFERENCES}

${ }^{1}$ Brass, L. F., and S. L. Diamond. Transport physics and biorheology in the setting of hemostasis and thrombosis. $J$. Thromb. Haemost. 14:906-917, 2016.

${ }^{2}$ Colace, T. V., G. W. Tormoen, O. J. T. McCarty, and S. L. Diamond. Microfluidics and coagulation biology. Annu. Rev. Biomed. Eng. 15:283-303, 2013.

${ }^{3}$ Fogelson, A. L., and K. B. Neeves. Fluid mechanics of blood clot formation. Annu. Rev. Fluid Mech. 47:377-403, 2015.

${ }^{4}$ Neeves, K. B., S. F. Maloney, K. P. Fong, A. A. Schmaier, M. L. Kahn, L. F. Brass, and S. L. Diamond. Microfluidic focal thrombosis model for measuring murine platelet deposition and stability: PAR4 signaling enhances shearresistance of platelet aggregates. J. Thromb. Haemost. 6:2193-2201, 2008.

${ }^{5}$ Schoeman, R. M., K. Rana, N. Danes, M. Lehmann, J. A. Di Paola, A. L. Fogelson, K. Leiderman, and K. B. Neeves. A microfluidic model of hemostasis sensitive to platelet function and coagulation. Cell. Mol. Bioeng. 2017. doi: 10.1007/s12195-016-0469-0.

${ }^{6}$ Zilberman-Rudenko, J., J. L. Sylman, H. H. S. Lakshmanan, O. J. T. McCarty, and J. Maddala. Dynamics of blood flow and thrombus formation in a multi-bypass microfluidic ladder network. Cell. Mol. Bioeng. 2017. doi: 10.1007/s12195-016-0470-7. 